This item has been removed at the request of the author and is no longer available on this site but can be accessed here:

http://www.enterprisingcompetencies.com/uploads/1/5/9/7/15971972/

vn_gelderen_2005_sbej_nascents.pdf 


\title{
Success and risk factors in the pre-startup phase
}

\author{
Van Gelderen, Marco
}

2006

http://hdl.handle.net/10179/9691

22/04/2023 - Downloaded from MASSEY RESEARCH ONLINE 